\title{
Background and bridge functions for the homonuclear hard diatomic fluid
}

Cite as: J. Chem. Phys. 90, 7330 (1989); https://doi.org/10.1063/1.456212

Submitted: 11 January 1989 . Accepted: 13 February 1989 . Published Online: 31 August 1998

E. Lomba, M. Lombardero, and J. L. F. Abascal

\section{ARTICLES YOU MAY BE INTERESTED IN}

Thermodynamics and simulation of hard-sphere fluid and solid: Kinetic Monte Carlo method versus standard Metropolis scheme

The Journal of Chemical Physics 146, 034110 (2017); https://doi.org/10.1063/1.4974141
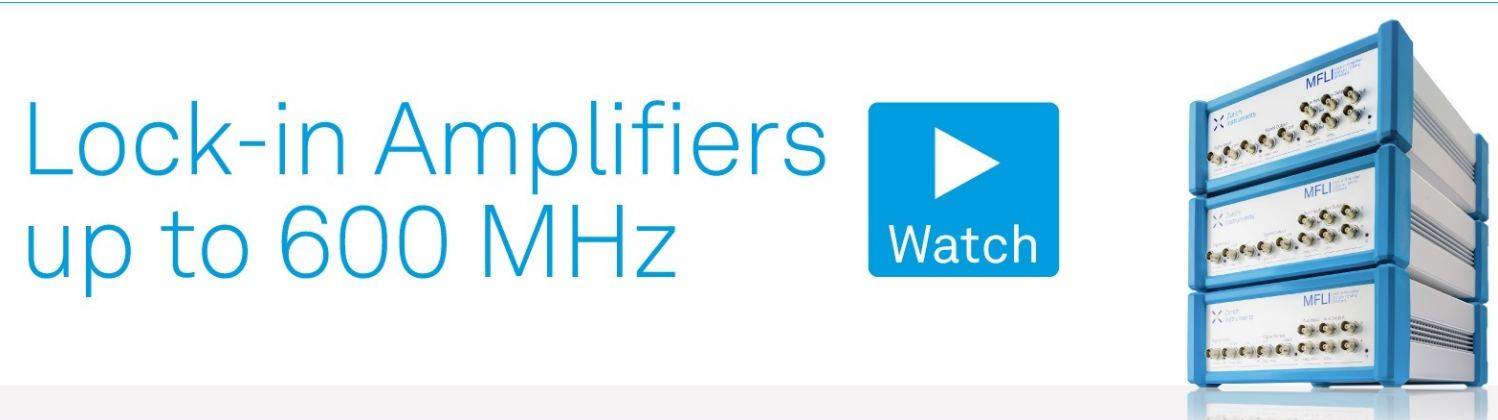


\title{
Background and bridge functions for the homonuclear hard diatomic fluid
}

\author{
E. Lomba and M. Lombardero \\ Instituto de Química Fisica Rocasolano, C.S.I.C., Serrano 119, E-28006 Madrid, Spain and Depto. \\ Química Fisica, Facultad de CC. Químicas Universidad Complutense de Madrid, E-28040 Madrid, Spain
}

J. L. F. Abascal

Depto. Química Fisica, Facultad de CC. Químicas, Universidad Complutense de Madrid, E-28040 Madrid, Spain

(Received 11 January 1989; accepted 13 February 1989)

The Monte Carlo method has been used to compute the coefficients of the spherical harmonic expansion of the function $y=g \exp (\beta u)$ for a hard diatomic fluid. The "series" function $S(12)$ is also computed from MC data by means of an integral equation procedure. Thus, the $B_{k l m}(r)$ terms of the harmonic series of the bridge function $B(12)$ can be easily obtained. The spherical harmonic expansion has proved an efficient tool to deal with these angular functions since the series is very rapidly convergent. We also have investigated the Percus-Yevick approximation both for the $S(12)$ and the $B(12)$, and a remarkable qualitative agreement with our MC data is found.

\section{INTRODUCTION}

The background correlation function (cavity distribution function) $y(12)$ as well as the bridge function $B(12)$ provide a very convenient mathematical tool for the development of efficient theories to deal with the structure and thermodynamics of fluids. Actually, they play an essential role in some perturbation and, especially, integral equation theories. However, it has been proven difficult to tackle the problem of computing such functions, either numerically or analytically, even in the simplest case of hard sphere (HS) fluids. In this regard, the pioneering work of Meeron and Siegert, ${ }^{1}$ reformulated in different ways, has led to several approaches to determine $\boldsymbol{y}(r)$ for the HS system. Grundke and Henderson ${ }^{2}$ have proposed an interpolation formula based on the knowledge of $y(r)$ at $r=0$ from thermodynamics, and Labík et al. ${ }^{3}$ and Torrie and Patey ${ }^{4}$ have presented the first computer simulation results for the background correlation function of the hard sphere fluid. More recently, Balance and Speedy ${ }^{5}$ performed molecular dynamic simulations for the hard disk fluid. Nonetheless, computations of $y(12)$ for nonspherical fluids are scarce in the literature. To our knowledge only Labík and Malijevsky ${ }^{3}$ carried out simulations for homonuclear hard diatomics (HOHD), and the same authors proposed an analytical approach, ${ }^{6}$ based on the use of the virial coefficients for infinitely dilute mixtures of an $n$-atomic fluid (solvent) and a $2 n$-atomic fluid (solute). Their work, however, is focused on the determination of radial slices through $y(12)$ and therefore their method does not seem suitable for an efficient computation of $y(12)$ at arbitrary interparticle orientations since the whole procedure must be repeated every time a new intermolecular configuration (even keeping the center-to-center distance constant) is required. In this sense, the use of the spherical harmonic expansion seems promising due to the expected regular behavior of $y(12)$ which implies that the knowledge of the coefficients $y_{k l m}(r)$ immediately furnishes the values of $y(12)$ for any given interparticle orientation by a simple numerical algorithm (series resummation).

Only little is known about the bridge function, and most of it for the hard sphere fluid. This function can be defined by means of a diagrammatic analysis ${ }^{7}$ and, since several of its graphs can be computed for hard spheres, it is possible to determine in this case the behavior of $B(r)$ in the low density region. ${ }^{8}$ On the other hand, at higher densities it can be computed via the Verlet and Weis parametrization of $g(r) .^{9,10}$ Recently, Malijevsky and Labík ${ }^{11}$ proposed an empirical formula based on the Percus-Yevick (PY) approximation whose results are consistent with computer simulation data for thermodynamic and structural properties. When considering nonspherical particles, the problem becomes even more involved. We are not aware of any work that have dealt with the determination of $B(\mathbf{1 2})$ in this sort of models; the difficulties in the computation of the background correlation function have hindered so far any attempt to extend the procedures available for the hard sphere fluid to the HOHD fluid. The bridge function in this fluids is, nevertheless, an interesting quantity since it can be used to extend some integral equation theories-in particular, the well known reference hypernetted chain equation ${ }^{12}$ (RHNC)-to nonspherical particles. Moreover, one may expect for this function a very regular behavior so that the effects of the anisotropy should be minimized. This would yield, as an immediate consequence, a rapid convergence in a spherical harmonic expansion.

In this work we present some results for the coefficients of the expansion of the background correlation function in spherical harmonics for the HOHD fluid, computed by means of a modification due to us of the method proposed by Labík et $a l^{2}$ We also introduce here some results for the bridge function. The method, fully explained in Sec. II, is based upon the relationship between the direct correlation and the bridge functions

$$
c(12)=h(12)-\ln [y(12)]-B(12),
$$

where $h(12)=g(12)-1$ is the total correlation function. This expression can be written in terms of the series function, $S(12)=h(12)-c(12)$, which is convenient from a numerical standpoint 


$$
B(12)=S(12)-\ln [y(12)] .
$$

$S(12)$ can be computed from the total correlation function $h(12)$ via the Ornstein-Zernike $(\mathrm{OZ})$ equation

$$
h(12)=c(12)+\left(\frac{\rho}{4 \pi}\right) \int c(13) h(32) d 3 .
$$

The fast convergence in the spherical harmonic expansions of $y(12)$ and $S(12)$ is the main characteristic that makes feasible this approach. The computation of the bridge function utilizing the aforementioned procedure will enable us to analyze from a new perspective widely used approximations to the solution of the $\mathrm{OZ}$ equation such as the Percus-Yevick and the hypernetted chain (HNC) equations.

\section{THE $y(12)$ AND B(12) FUNCTIONS FROM MC SIMULATION}

The computational scheme implies two different but closely related aspects as one may see in Eq. (2). First, the spherical harmonic coefficients of the $y(12)$ function in a hard diatomic fluid can only be determined with some reliability using Monte Carlo (MC) simulation (or some theoretically supported approximation which, in the end, will have to be tested against MC data). At the same time one must compute the $S(12)$ function (or its spherical harmonic coefficients), either from some theoretical approach or using $\mathrm{MC}$ data for the pair distribution function $g(12)$. Once these two steps have been accomplished, the determination of $B(12)$ [or more precisely the coefficients $B_{k l m}(r)$ ] is straightforward. In what follows we will explore the different possibilities available to tackle the two main problems we have mentioned, both in the simulation and in the theoretical approach.

\section{A. MC simulation of the background correlation function $y(12)$}

In this subsection we shall recall the main features of the background function theory, within the Meeron and Siegert approach. ${ }^{1}$ The numerical method sketched below, essentially devised by Labík et al., ${ }^{3}$ has been modified by us so that it yields the spherical harmonic coefficients. It is worth noticing that the main advantage in this procedure lies in the considerable saving of computing time, its principal shortcoming being its restriction to hard core potentials and that the accuracy noticeably decreases with distance and density. On the contrary, the Torrie and Patey procedure, ${ }^{4}$ even though the computations can be extremely tedious, is not constrained to hard core interactions. Since as yet we are only interested in the hard diatomic fluid, the Labík and Malijevsky procedure seems more adequate for low and medium densities. An alternative route should be necessary if some additional contributions-e.g., electrostatic interactions-were to be added to the hard core term. by

The background correlation function $y(12)$ is defined

$$
y(12)=\exp [\beta u(12)] g(12)
$$

from which, using the definition of $g(\mathbf{1 2})$ and assuming the potential $U_{N}(12 \cdots \mathrm{N})$ to be pairwise additive, one may get ${ }^{3}$

$$
\begin{gathered}
y(12)=\frac{V^{2}}{Z_{N}} \int \cdots \int \prod_{j>1} e(1 \mathbf{j}) \prod_{j>2} e(2 \mathbf{j}) \\
\times \prod_{i>j>2} e(\mathbf{i j}) d \mathbf{3} \cdots d \mathbf{N} \\
Z_{N}=\int \cdots \int \prod_{i>j>1} e(\mathbf{i j}) d \mathbf{1} \cdots d \mathbf{N}
\end{gathered}
$$

where $e(\mathbf{i j})=\exp [-\beta u(\mathbf{i j})]$. In order to get some physical insight from Eq. (5), Meeron and Siegert introduced the concept of cavity when dealing with the $y(12)$ function: a domain within a fluid where no particle is present. This definition implies that whereas there is no interaction between two cavities, cavities and molecules can not overlap in a hard spheres or hard diatomics fluid. The probability of finding two of such cavities at positions $r_{1}$ and $r_{2}$, and orientations $\omega_{1}$ and $\omega_{2}$, respectively, in a system of $N-2$ hard diatomics is given by ${ }^{13}$

$n_{N}^{(2)}(12)$

$$
\begin{aligned}
= & \left(V^{2} \int \cdots \int \prod_{i>2} e(\mathbf{1 i}) \prod_{j>2} e(2 \mathbf{j}) \prod_{j>i>2} e(\mathbf{i j}) d 3 \cdots d N\right) / \\
& \left(\iint \prod_{i>j>2} e(\mathbf{i j}) d 3 \cdots d \mathbf{N}\right),
\end{aligned}
$$

an expression closely related to Eq. (5); hence the name "cavity distribution function" for the $y(12)$. In fact, Eq. (6), after some manipulations, leads to the following relation:

$$
y(12)=V^{2}\left(Z_{N-1} / Z_{N}\right) n_{N}^{(2)}(12) .
$$

From elementary statistical thermodynamics one may get $^{13}$

$$
\beta \Delta \mu=-\ln \left(Z_{N} / Z_{N-1} V\right)
$$

from which Eq. (6) in the thermodynamic limit yields

$$
y(12)=\exp (2 \beta \Delta \mu) n_{N}^{(2)}(12),
$$

where $\Delta \mu$ is the excess chemical potential of the HOHD fluid. From this result Hoover and Poirier, ${ }^{14}$ and later Meeron and Siegert ${ }^{1}$ showed that

$$
\begin{aligned}
\lim _{r_{12} \rightarrow 0} y\left(r_{12}, \omega_{1}, \omega_{2}\right) \\
=\exp [\beta \Delta \mu], \text { if and only if } \omega_{1}=\omega_{2} .
\end{aligned}
$$

This expression plays a central role in the development of the computer simulation method used to calculate $y(12)$. Following Labík et al. [Eq. (5) in Ref. 3]

$$
\begin{aligned}
\lim _{r_{12} \rightarrow 0} y\left(r_{12}, \omega, \omega\right) \\
=\frac{V^{2}}{Z_{N}} \int \cdots \int \prod_{j>2} e(2 \mathbf{j}) \prod_{i>j>2} e(\mathbf{i j}) d \mathbf{3} \cdots d \mathbf{N} .
\end{aligned}
$$

In the above expression it has been taken into account that for hard core potentials $e(\mathbf{i j})^{2}=e(\mathbf{i j})$, since $e(\mathbf{i j})$ is either zero or unity. By using Eqs. (5), (10), and (11) the following function may be defined:

$$
P\left(r_{12}, \omega_{1}, \omega_{2}\right)=\exp (-\beta \Delta \mu) y\left(r_{12}, \omega_{1}, \omega_{2}\right)
$$

which is equivalent to 


$$
\begin{aligned}
& P\left(r_{12}, \omega_{1}, \omega_{2}\right) \\
& \quad=\frac{1}{Z_{N}} \int \cdots \int \prod_{j>2} e(\mathbf{i j}) \prod_{i>j>1} e(\mathbf{i j}) d 3 \cdots d \mathbf{N} .
\end{aligned}
$$

From this expression one may conclude that $P\left(r_{12}, \omega_{1}, \omega_{2}\right)$ represents the probability of inserting a new cavity at $\mathbf{r}_{1}$ and orientation $\omega_{1}$ (cavity 1 ) in a sample composed of $N-1$ particles and a cavity whose coordinates are $\left(\mathbf{r}_{2}, \omega_{2}\right)$ (cavity 2$)$. Obviously, the new cavity can not be inserted if it overlaps any molecule in the sample. This procedure bypasses the difficulty-unavoidable in the Torrie and Patey procedure-in simulating a sample with two cavities plus $N$-2 particles. Actually, one needs only to consider the sample configurations of an $N$-particle system and compute the probability of inserting a single cavity 1; any of the $N$ particles can be regarded as the cavity 2 since a system formed by $N$ nonoverlapping particles is completely equivalent to $N-1$ particles plus a cavity. This implies that a standard Monte Carlo programme for hard core particles can be used to generate the configurations, thus allowing a considerable saving of computing time and improving the reliability of the results, as $N$ equivalent attempts of insertion can be performed.

The spherical harmonic coefficients can be obtained during the insertion procedure once the probability for a given interparticle configuration-described by $\left(r_{12}, \omega_{1}, \omega_{2}\right)$-has been computed. The method consists simply in projecting the $P\left(\mathrm{r}_{12}, \omega_{1}, \omega_{2}\right)$ function onto the appropriate products of spherical harmonics $Y_{k m}\left(\omega_{1}\right) Y_{l \bar{m}}\left(\omega_{2}\right)$ and compute the angular average over $\omega_{1}$ and $\omega_{2}$. Numerically, this reduces to chose a random orientation at a given center to center distance, $r_{12}$ - the orientation of $\mathbf{r}_{12}$ being randomly selected as well-and perform the insertion attempt. This procedure must be carried out for every molecule and sample configuration. In this way the spherical harmonic coefficients of the $P\left(\mathbf{r}_{12}, \omega_{1}, \omega_{2}\right)$ can be expressed as

$$
\begin{aligned}
P_{k l m}\left(r_{12}\right)= & \frac{1}{N_{c}} \sum_{j}^{N_{c}} \frac{1}{n_{a}} \cdot \sum_{i}^{n_{a}} p_{i}^{j}\left(r_{12}, \omega_{1_{i}}^{j}, \omega_{2_{i}}^{j}\right) \\
& \times Y_{k m}\left(\omega_{i_{i}}^{j}\right) Y_{l m}\left(\omega_{2_{i}}^{j}\right),
\end{aligned}
$$

where $p_{i}^{j}\left(r_{12}, \omega_{1_{i}}^{j}, \omega_{2_{i}}^{j}\right)$ is a step function whose value is either zero or unity depending on whether the attempt of insertion was successful or not, and the index $i$ stands for the $i$ th attempt of insertion for the state $j ; N_{c}$ is the number of configurations and $n_{a}$ the number of tentatives-at least one per particle. Using Eq. (12) we get the following formula for the $y_{k l m}(r)$ coefficients

$$
\begin{aligned}
y_{k l m}\left(r_{12}\right)= & \exp (\beta \Delta \mu) \frac{1}{N_{c}} \sum_{j}^{N_{c}} \frac{1}{n_{a}} \cdot \sum_{i}^{n_{a}} p_{i}^{j}\left(r_{12}, \omega_{1_{i}}^{j}, \omega_{2_{i}}^{j}\right) \\
& \times Y_{k m}\left(\omega_{1_{i}}^{j}\right) Y_{l \bar{m}}\left(\omega_{2_{i}}^{j}\right) .
\end{aligned}
$$

The excess chemical potential has been calculated using the Nezbeda and Boublik equation of state ${ }^{15}$

$$
\frac{\beta p}{\rho}=\frac{1+(3 \alpha-2) \eta+\left(3 \alpha^{2}-3 \alpha+1\right) \eta^{2}-\alpha^{2} \eta^{3}}{(1-\eta)^{3}}
$$

with

$$
\begin{aligned}
& \alpha=\left(l^{*}+1\right)\left(l^{*}+2\right) /\left(2+3 l^{*}-l^{* 3}\right), \\
& \eta=(\pi / 6) \rho d^{3}\left(1+3 l^{*} / 2-l^{* 3} / 2\right),
\end{aligned}
$$

where $l^{*}=l / d$, with $l$ being the center-to-center separation of the atomic sites in the diatomic molecules and $d$ is the atomic diameter. Integrating $\mathrm{Eq}$. (16) the final expression for the excess chemical potential reduces to

$$
\begin{aligned}
\beta \Delta \mu= & \frac{\left(\alpha^{2}-3 \alpha\right) \eta-3 \alpha \eta^{2}}{(1-\eta)^{2}} \\
& -\left(1-\alpha^{2}\right) \ln (1-\eta)+\frac{\beta p}{\rho}-1 .
\end{aligned}
$$

This relation was found to be accurate enough to our purposes as we shall see in Sec. IV.

Once the $y_{k l m}(r)$ coefficients have been computed, the full $y(12)$ function must be determined by resummation of the spherical harmonic expansion, so that Eq. (2) can be used to obtain $B(12)$. One easily may get

$$
y\left(r_{12}, \omega_{1}, \omega_{2}\right)=4 \pi \sum_{k l m} y_{k l m}\left(r_{12}\right) Y_{k m}\left(\omega_{1}\right) Y_{l \bar{m}}\left(\omega_{2}\right)
$$

for any given set $\left(r_{12}, \omega_{1}, \omega_{2}\right)$. On the contrary, the Labík and Malijevsky original method $^{3}$ based on the determination of the full $y(12)$ for given configurations, would imply the use of interpolation procedures.

\section{B. Calculation of the series function S(12)}

In order to obtain $S(12)$ from the simulated spherical harmonic coefficients of $g(12)$, the $O Z$ equation must be inverted, using $h(12)$ as the input function. Previous works $^{11,16}$ suggest that the inversion of the $\mathrm{OZ}$ equation from simulation data is numerically unreliable since it tends to enhance inaccuracies when $S(12)$ is expressed in terms of $h(12)$. Moreover, the values of $h(\mathbf{1 2})$-or alternatively the coefficients $h_{k l m}(r)$-are obtained in the simulation within a limited range of $r$ determined by the size of the unit cell, namely $r<R_{m}$, where $R_{m}=L / 2$ ( $L$ being the side of the cell) and, since the inversion of the $\mathrm{OZ}$ is performed in the Fourier space, the knowledge of $h\left(r_{12}, \omega_{1}, \omega_{2}\right)$ for larger values of $r_{12}$ would be required. Therefore, we use a procedure found in the literature-although in a different context ${ }^{17}$ to bypass similar difficulties. As a first step one should rewrite the $\mathrm{OZ}$ equation in terms of $S(12)$

$$
S(12)=\left(\frac{\rho}{4 \pi}\right) \int[S(13)+c(13)] c(32) d 3
$$

The $\mathrm{OZ}$ equation expressed in this way is more suitable for numerical computations, ${ }^{12}$ especially when dealing with nonspherical problems due to the rapid convergence of the $c(12)$ and $S(12)$ expansions. If we denote by $h^{\mathrm{MC}}(12)$ the simulation results for the total correlation function, we can set up the following closure relation for Eq. (20):

$$
\begin{aligned}
& c(12)=h^{\mathrm{MC}}(12)-S(12) \quad \text { if } r \leqslant R_{m}, \\
& c(12)=0 \quad \text { if } r>R_{m},
\end{aligned}
$$

where obviously Eq. (21a) is exact within the statistical uncertainties of the simulation and Eq. (21b) is an approxima- 
tion. Clearly, relation (21) resembles the PY approximation but can be regarded as quasiexact since $R_{m} \sim 4 \sigma$ ( $\sigma$ being the atomic diameter) for a sample of 256 particles at liquid densities, distance for which any hard core potential would render a vanishing $c(\mathbf{1 2})$.

Now, the problem of computing the series function $S(12)$ reduces to solving Eqs. (20) and (21) in terms of the spherical harmonic coefficients. This can be handled by means of a procedure devised by Lado, ${ }^{12}$ which we briefly outline below.

In Eq. (20) we may expand both $S(12)$ and $c(12)$ by which, using the orthogonality of the spherical harmonics, one gets

$$
\begin{aligned}
S_{k l m}\left(r_{12}\right)= & (-1)^{m} \rho \cdot \sum_{\lambda} \int\left[S_{k \lambda m}\left(r_{13}\right)+c_{k \lambda m}\left(r_{13}\right)\right] \\
& \times c_{\lambda l m}\left(r_{32}\right) d \mathbf{r}_{3} .
\end{aligned}
$$

This expression is equivalent to a matrix equation where $(\mathrm{kl})$ are the element index, and the dimensionality of the matrix is given by the maximum value of $m$ to be considered. Obviously, the values of $k$ and $l$ must be compatible with $m$, i.e., $k, l \geqslant m$. Thus, by means of Fourier transforms (below denoted by a tilde) and Eq. (22), the $\tilde{S}_{k l m}(k)$ can be expressed as

$$
\begin{aligned}
k \tilde{S}_{m}(k)= & (-1)^{m} \rho\left\{k \mathbf{I}-(-1)^{m} \rho\left[k \tilde{\mathbf{c}}_{m}(k)\right]\right\}^{-1} \\
& \times\left[k \tilde{\mathbf{c}}_{m}(k)\right]^{2} .
\end{aligned}
$$

The computation of these Fourier transforms is by no means straightforward. We have used an efficient algorithm proposed by Lado which can be found fully explained in Ref. 12 .

In this way, once an initial solution for the $S_{k l m}(r)$ coefficients is available (e.g., the hard spheres one), after insertion in Eqs. (21) one can get $c_{k l m}(r)$. These coefficients are Fourier transformed as indicated above, and a new set of $S_{k l m}(r)$ coefficients is obtained from Eq. (23), tranforming back to real space. In order to assure convergence it is advisable the utilization of a Broyles mixing iterates method ${ }^{18}$ at high densities. An adequate convergence criterion is the one suggested by Lado ${ }^{12} r\left|S_{k l m}(r)-S_{k l m}(r)\right|<10^{-3}$ [usually the fulfillment by $S_{000}(r)$ implies the convergence of the whole set of harmonic coefficients].

\section{Computation of the bridge function coefficients}

In Secs. II A and II B we have explained the procedures to calculate the $y(12)$ and $S(12)$ functions from MC data. Now one may easily perform the computation of $B_{k l m}(r)$ from Eq. (2) expressed in terms of spherical harmonic coefficients

$$
\begin{aligned}
B_{\lambda \mu \nu}(r) & \\
= & S_{\lambda \mu \nu}(r)-\left\langle\ln \left[4 \pi \sum_{k l m} y_{k l m}(r) Y_{k m}\left(\omega_{1}\right) Y_{l \bar{m}}\left(\omega_{2}\right)\right]\right. \\
& \left.\times Y_{\lambda \nu}\left(\omega_{1}\right) Y_{\mu \bar{\nu}}\left(\omega_{2}\right)\right\rangle,
\end{aligned}
$$

where $\langle\cdots\rangle$ denotes angular average over orientations $\omega_{1}$ and $\omega_{2}$, and all the terms on the right-hand side of Eq. (24) should have been previously computed. It must be men- tioned that in the region $r \geqslant \sigma+1 y_{k l m}(r)=g_{k l m}(r)$, and therefore it is advisable the use of the latter coefficients because of their better statistics. In this equation, the presence of the logarithmic term minimizes the inaccuracies in $y$ (12) which highly increase with density.

\section{SOME THEORETICAL APPROACHES}

With regard to theoretical approximations to the bridge function, we should mention the Percus-Yevick (PY) and the hypperneted chain (HNC). These are widely used closure relations in the context of the solution of the $\mathrm{OZ}$ equation. One of them, the HNC in fact reduces to assume the bridge function to be identically zero for all the values of $r$. This approximation is known to yield rather good results when dealing with the long range properties of fluids. On the other hand, the PY approximation, whose efficiency for short range potentials is well established, assumes a simple relation between $y(12)$ and $S(12)$, such as

$$
y(12)=1+S(12)
$$

which, in turn, gives the following expression for the bridge function:

$$
B(12)=S(12)-\ln [1+S(12)]
$$

When Eq. (25) is expressed in terms of $g(12)$ and $S(12)$ one gets the usual PY closure relation for $g(12)$

$$
\begin{aligned}
& g(12)=[1+S(12)] \exp [-\beta u(12)] \\
& c(12)=[1+S(12)]\{\exp [-\beta u(12)]-1\}
\end{aligned}
$$$$
\text { or }
$$

in terms of $c(12)$. Thus, it is possible to solve the $\mathrm{OZ}$ equation [Eq. (20)] to obtain $S(12)$ and insert this solution into Eq. (26); this would lead to the Percus-Yevick approximation for the bridge function. In order to solve the $\mathrm{OZ}$ equation with the PY closure relation for the hard diatomic fluid, the procedure sketched in Sec. II B has been applied. The only difference lies in the fact that now the relation between $c(12)$ and $S(12)$ is nonlinear; thereby the projection of $c$ (12) [Eq. (28)] onto $Y_{k m}\left(\omega_{1}\right) \cdot Y_{l \bar{m}}\left(\omega_{2}\right)$ must be explicitly computed through

$$
\begin{aligned}
c_{k l m}(r)= & \frac{1}{4 \pi} \iint[1+S(12)]\{\exp [-\beta u(12)]-1\} \\
& \times Y_{k \bar{m}}\left(\omega_{1}\right) Y_{l m}\left(\omega_{2}\right) d \omega_{1} d \omega_{2} .
\end{aligned}
$$

Note that in the case of Eq. (21) the projection is trivial. A Broyles method ${ }^{18}$ is required to assure a satisfactory rate of convergence in the $S_{k l m}(r)$ coefficients.

\section{NUMERICAL RESULTS}

We have carried out Monte Carlo simulations for a hard diatomic fluid with elongation $l=0.6 \sigma$ at three different densities $\rho \sigma^{3}=0.2,0.3$, and 0.4352 using 256 particles. In what follows and in the figures we will use the convention $\rho \sigma^{3}=\rho^{*}$ and $r / \sigma=r^{*}$ as usual. The averages in the simulation were computed every 1500 sample configurations and the total number of configurations ranged from $2.5 \times 10^{6}$ to 
$4.3 \times 10^{6}$, depending on density. From these simulations we first obtained the $g_{k l m}(r)$ coefficients, which we do not include in this work since they are well known quantities for this particular model. ${ }^{19}$ The same configurations were used to compute the $y_{k l m}(r)$; following the method explained in the previous section we have carried out two insertion attempts per sample particle at the lowest density, three attempts at $\rho^{*}=0.3$ and six at the highest density, $\rho^{*}=0.4352$. Since the quality of the results depends upon the probability of cavity insertion, simulations for high densities are far more tedious than those for low ones. In practice, this insertion procedure becomes useless for densities higher than those considered here since the computing time rises exponentially with the density.

A check of our results is shown in Fig. 1, where the first two coefficients $y_{k l m}(r)$ (black and white triangles) at $\rho^{*}=0.4352$ are represented vs the results obtained for the $g_{k l m}(r)$ (solid lines). It must be recalled that, since we are dealing with a homonuclear hard diatomic fluid, $g_{k l m}(r)$ $=y_{k l m}(r)$ for $r \geqslant l+\sigma$. One may immediately see from the figure that lines and dots in this region show a remarkable agreement, at least within the inaccuracies of $y(12)$ in the MC simulation.

In Fig. 2, the most significant coefficients of the $y(12)$ spherical harmonic expansion are presented. First, it should be noticed the sharp decrease in the magnitude of the $y_{k l m}(r)$ coefficients as the $k l$ indexes increase. This obviously implies a fast convergence of the expansion. Besides, the $r$ dependence of all the coefficients shows an outstanding regular behavior, and since they reach the largest values in the vicinity of $r=0$, one may conclude that this is the zone where the angular dependence of $y(\mathbf{1 2})$ is more significant. A curious feature one can appreciate in Fig. 2 is that the angular dependence is determined mainly by the coefficients $y_{l l m}(r)(000$, $22 m, 44 m$ ) which show a quite symmetric distribution with regard to the $y_{k l m}(r)=0$ axis.

In order to confirm the rapid convergence of the $y(12)$ expansion, in Fig. 3 we present the values of this function at $\rho^{*}=0.3$ computed for several intermolecular configura-

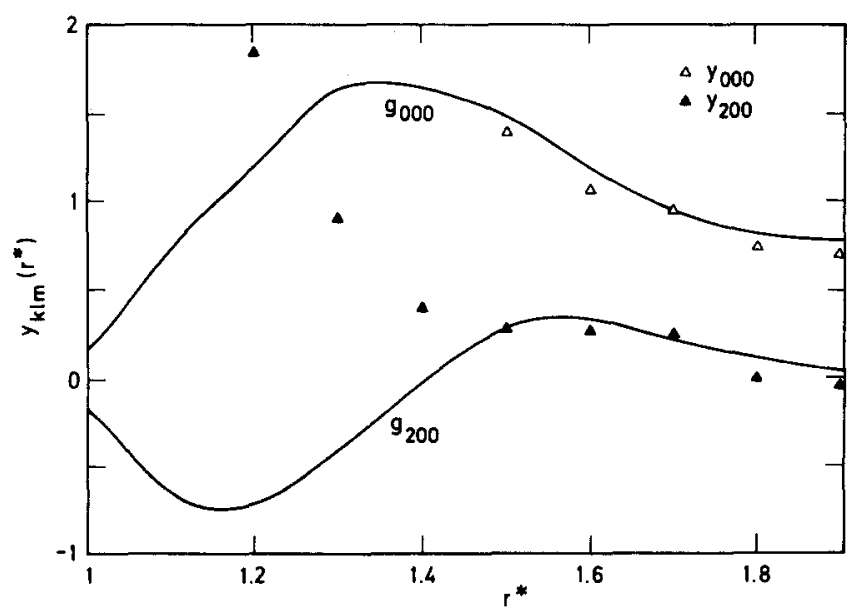

FIG. 1. First coefficients of the spherical harmonic expansion of the $g(12)$ (solid lines) and the $y(12)$ function (triangles) computed at $\rho^{*}=0.4352$.

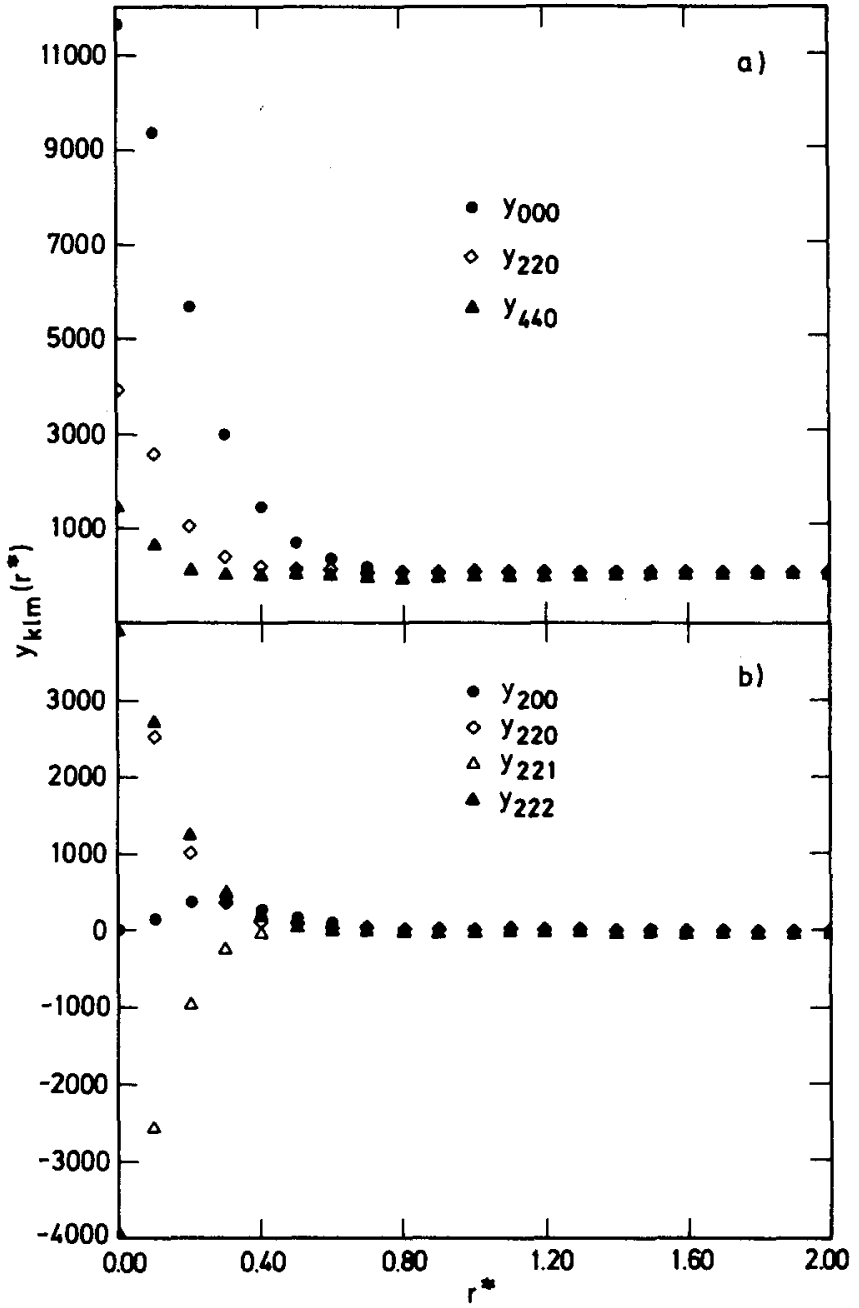

FIG. 2. Most significant coefficients of the $y(12)$ expansion at $\rho^{*}=0.4352$.



FIG. 3. The function $\ln [y(12)]$ computed for several intermolecular configurations at $\rho^{*}=0.3$, namely, end-to-end (e-e), T-shaped (T), parallel $(=)$, and crossed $(X)$ in this work (triangles and squares) and in Ref. 3. 
tions (end-to-end, T-shaped, parallel, and crossed) via resummation of the expansion (up to $y_{444}$ ), compared with those obtained directly by Labík et al. ${ }^{3}$ The agreement between both results is clear and needs no further explanation. The examination of Figs. 2 and 3 guarantees that an accurate computation of the value of $y\left(r_{12}, \omega_{1}, \omega_{2}\right)$ at any given intermolecular configuration is attainable by resummation of the spherical harmonic expansion. This is an important conclusion since it is one of the properties that enable us the determination of the bridge function via Eq. (24).

In Fig. 4 one may observe the change induced in the $y_{k l m}(r)$ when density is increased, both in the radial term and in the angular coefficient $\left(y_{200}\right)$. The density dependence exhibited by both coefficients is easily understood if one recalls Eq. (12), where the $\rho^{*}$ dependence in $\Delta \mu$ is enhanced by the exponential. Moreover, the angular dependence of $P\left(r_{12}, \omega_{1}, \omega_{2}\right)$ is also augmented as density raises. In fact, at very high densities, $P\left(0, \omega_{i}, \omega_{j}\right)=1$, if $\omega_{i}$ is extremely close to $\omega_{j}$, and vanishes otherwise.

The $S_{k l m}(r)$ coefficients of the series function were obtained by means of the procedure described in Sec. II B using MC data for $h_{k l m}(r)=g_{k l m}(r)-1$. The number of iterations required by our procedure ranges from 10 up to 40 (at

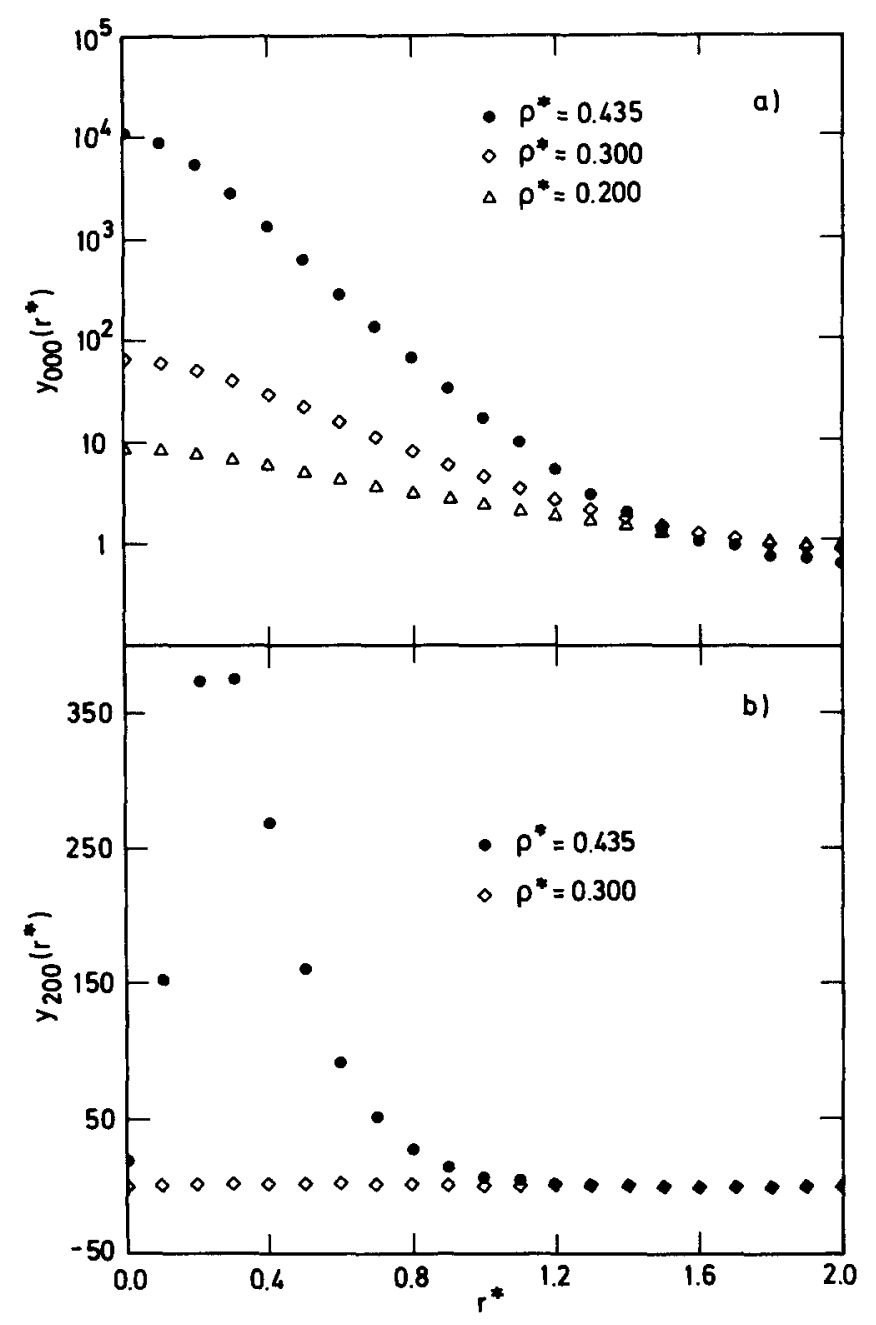

FIG. 4. The density dependence of the $y_{000}(r)$ and $y_{200}(r)$. Note that a log scale is used in (a). $\rho^{*}=0.4532$ ) and the convergence was achieved without special difficulties. We also solved the PY equation [Eqs. (22) and (28) ]. The angular averaging implied in Eq. (29) was performed using the procedure suggested by Lado, ${ }^{12}$ based on the transformation of the average into an integral suitable for the use of gaussian integration. The results are very sensitive to the number of points (in this case 30 points per angular variable sufficed). When inverting the $\mathrm{OZ}$ equation and solving the PY equation the Fourier transforms were performed using 512 points in $r$, with a spacing $\Delta r=0.02 \sigma$. The use of a larger number of points or a smaller grid does not alter significantly the results. In both cases the number of $S_{k l m}(r)$ coefficients computed is $15(k, l \leqslant 4)$. In this regard, Lado ${ }^{12}$ has shown that even the use of five coefficients $(k, l \leqslant 2)$ suffices in order to obtain an adequate representation of the function.

The most significant coefficients are shown in Fig. 5 for $\rho^{*}=0.4352$, where the symbols denote results obtained from MC data and lines indicate PY results. It is to be noticed the qualitative agreement between $\mathrm{MC}$ and PY results, both in the radial coefficient $S_{\mathrm{OoO}}(r)$ and in the angular projections $S_{21 m}(r)$. Conversely, if one compares the MC coefficients for $y(12)$ (see Fig. 2) with those obtained for $S(12)$ in

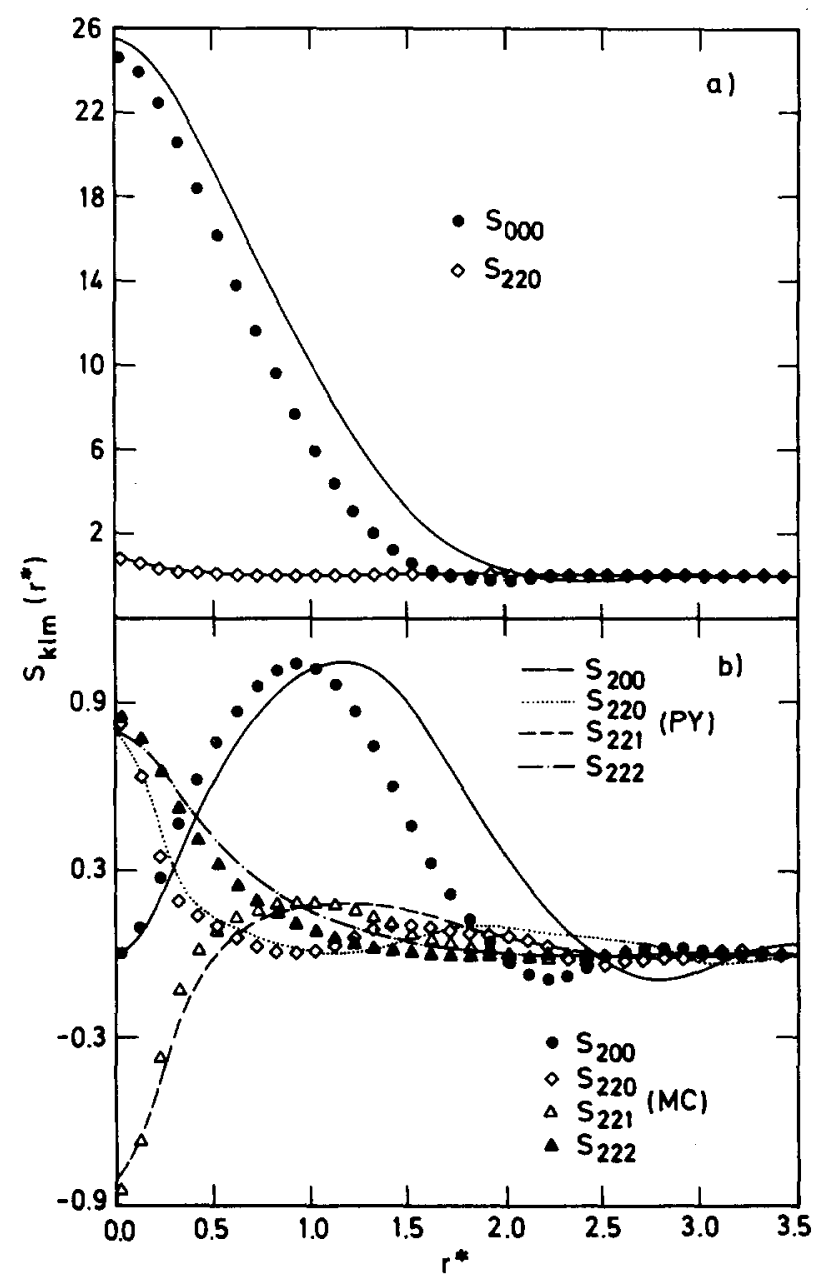

FIG. 5. Leading coefficients in the expansion of $S(12)$ at $\rho^{*}=0.4352$. Lines stand for the results of the PY approximation and symbols represent results obtained from $\mathrm{MC}$ data. 
the PY approximation [in which $y(12)=1+S(12)$ and thus $S_{k l m}(r)=y_{k l m}(r)$ if $(k l m) \neq(000)$ ], the discrepancies are considerable.

Once the $y_{k l m}(r)$ and $S_{k l m}(r)$ coefficients have been determined the computation of $B_{k l m}(r)$ is straightforward via Eq. (24)

The main results for the $B_{k l m}(r)$ coefficients are collected in Figs. 6 and 7. In Fig. 6 we have shown the density dependence of the radial coefficient in the same way we did in Fig. 4 with the projections of $y(12)$. The extreme density dependence of $y_{000}(r)$ is somehow damped by the logarithm in Eq. (24), in such a way that even though there is a remarkable increase in $B_{000}(r)$ with density it does not affect the order of magnitude, as it happens with $y_{000}(r)$. This effect can also be observed in the angular coefficients [Fig. 6(b) ], so that $S_{k l m}(r)$ are much less sensitive to density increments that the $y_{k l m}(r)$.

Finally, in Fig. 7 the most representative coefficients at $\rho^{*}=0.4352$ are depicted. As in Fig. 5, lines of different type represent PY approximation and symbols denote the results coming from MC data. With regard to our $\mathrm{MC}$ results it has to be stressed once more the rather regular behavior of the coefficients, as well as the rapid convergency of the $B(12)$ expansion. We are, however, aware of an evident deviation

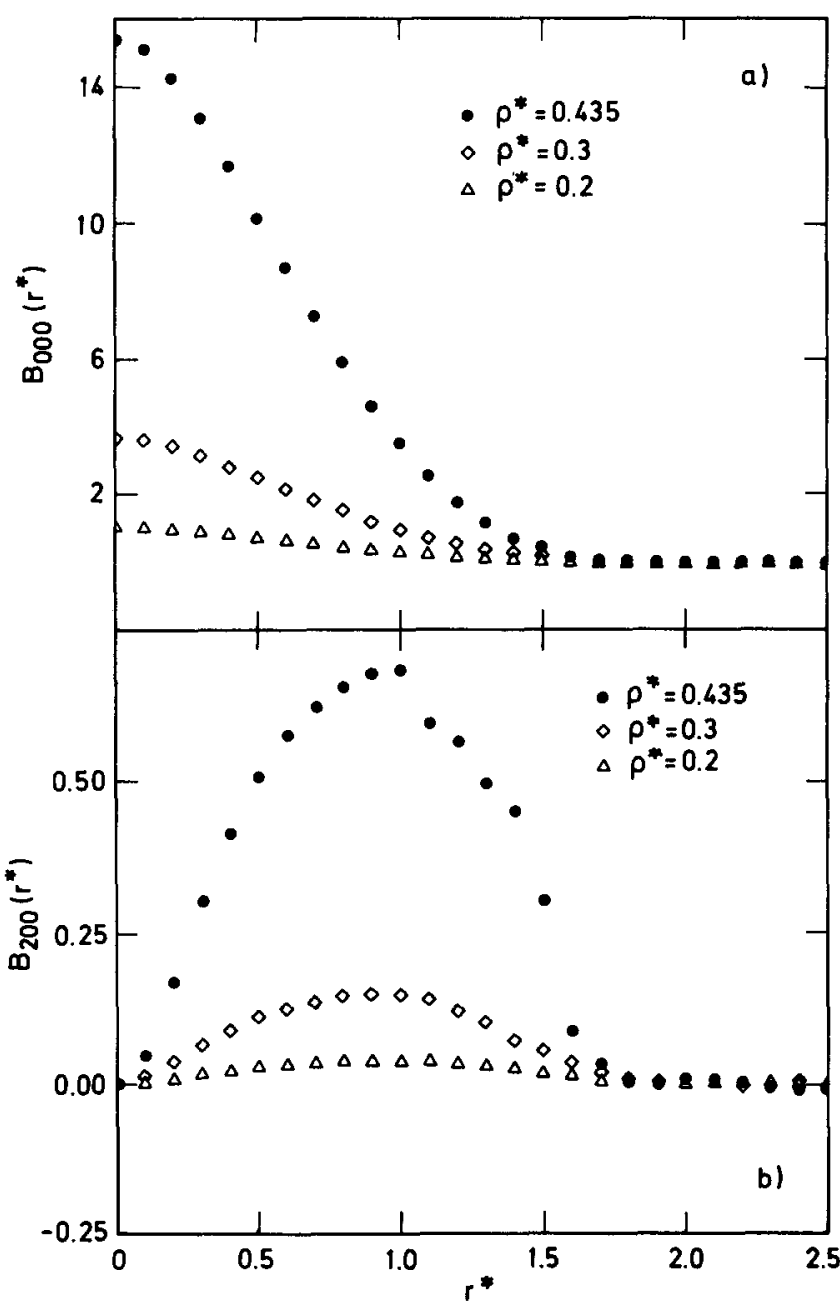

FIG. 6. The density dependence of the coefficients $B_{000}(r)$ and $B_{200}(r)$.

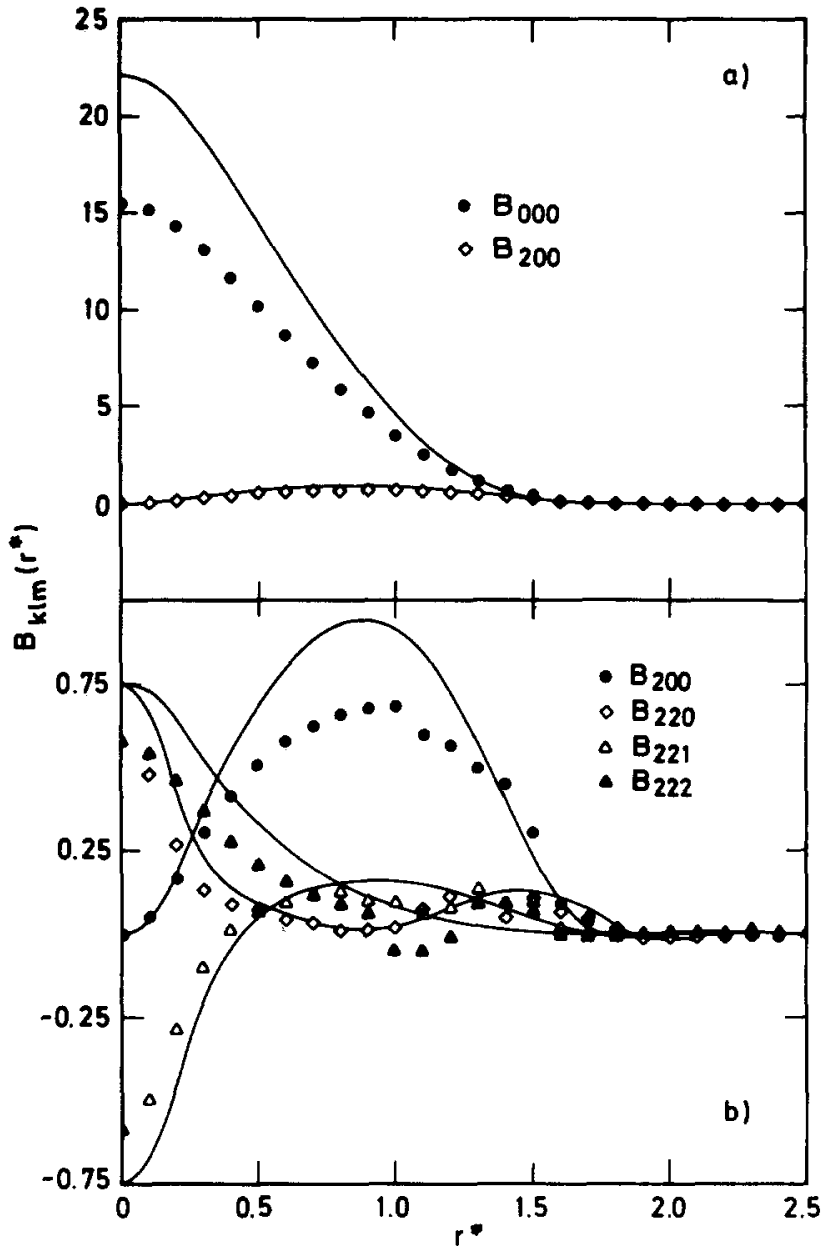

FIG. 7. Leading coefficients in the expansion of $B(12)$ at $\rho^{*}=0.4352$. Lines stand for the results of the PY approximation and symbols represent results obtained from MC data.

from this regular behavior in the region $\sigma<r<\sigma+l$, which is not present in the PY results. To our opinion this effect may stem from the statistical uncertainties in the MC simulation of $y(12)$ which render the $y_{4 l m}(r)$ coefficients almost meaningless for $r>\sigma$. These coefficients cannot be neglected in order to get an accurate determination of $\ln [y(12)]$ in Eq. (24) and, subsequently, this could give rise to the aforementioned deviations. In this regard, a similar phenomenon is found when the $g(12)$ is rebuilt from its spherical harmonic expansion in the same region. ${ }^{19}$ Finally, it should be mentioned that all the coefficients are relatively short ranged in $r$ [shorter than the $g_{k l m}(r)$ ], as it could be expected, $B(12)$ being a sum of convolutions of $h(12) .^{7}$

Again the PY results show a qualitative agreement with simulation data. Perhaps the most meaningful discrepancy appears in the value of $B_{000}(r)$ at $r=0$. This agreement is an interesting feature, in particular if one realizes that an approximation such as the HNC, simply neglects the contribution of all the $B_{k l m}(r)$ coefficients.

The qualitative agreement between $\mathrm{PY}$ and $\mathrm{MC}$ results, especially as far as the $B(12)$ function is concerned, may be of interest in order to extend fitting procedures, such as the one by Verlet and Weis, ${ }^{9}$ so that the bridge function could be 
determined in an easy and straightforward way for hard diatomic fluids.

\section{ACKNOWLEDGMENTS}

The authors wish to thank the CSIC and Universidad Complutense Computer Centres for access generously provided to their computing facilities. This study was supported by Grant SEUI No. PB87-0246-00 furnished by the Dirección General de Investigación Científica y Tecnológica of Spain.

'E. Meeron and A. J. F. Siegert, J. Chem Phys. 48, 3139 (1968).

${ }^{2}$ E. W. Grundke and D. Henderson, Mol. Phys. 24, 269 (1972).

${ }^{3}$ S. Labik and A. Malijevsky, Mol. Phys. 53, 381 (1984).
${ }^{4}$ G. Torrie and G. N. Patey, Mol. Phys. 34, 1623 (1977).

5J. A. Balance and R. J. Speedy, Mol. Phys. 54, 105 (1985).

'S. Labík and A. Malijevsky, Mol. Phys. 60, 1107 (1987).

'G. Stell, in The Equilibrium Theory of Classical Fluids, edited by H. L.

Frisch and J. L. Lebowitz (Benjamin, New York, 1964), p. II-171.

${ }^{\mathrm{x}}$ F. H. Ree, R. N. Keeler, and S. L. McCarthy, J. Chem. Phys. 44, 3407 (1966).

${ }^{9}$ L. Verlet and J. J. Weiss, Phys. Rev. A 5, 939 (1972).

1"D. Henderson and E. W. Grundke, J. Chem. Phys. 63, 601 (1975).

"A. Malijevsky and S. Labík, Mol. Phys. 60, 663 (1987).

${ }^{12}$ F. Lado, Mol. Phys. 47, 283, 299 (1982).

${ }^{13}$ T. Boublik, Mol. Phys. 59, 775 (1986).

${ }^{14}$ W. G. Hoover and J. C. Poirier, J. Chem. Phys. 48, 3139 (1963).

${ }^{15}$ T. Boublik and I. Nezbeda, Chem. Phys. Lett. 46, 315 (1977).

${ }^{16}$ F. Lado, J. Chem. Phys. 47, 4828 (1968).

${ }^{17}$ G. N. Patey, D. Levesque, and J. J. Weis, Mol. Phys. 45, 733 (1982).

${ }^{18}$ A. Broyles, J. Chem. Phys. 33, 456 (1960).

${ }^{10}$ W. B. Street and D. J. Tildesley, Proc. R. Soc. London Ser. A 348, 485 (1976). 